\title{
Rocking convex array used for 3D synthetic aperture focusing
}

\author{
Andresen, Henrik; Nikolov, Svetoslav; Pedersen, M M; Buckton, D; Jensen, Jørgen Arendt
}

Published in:

2008 IEEE Ultrasonics Symposium

Link to article, DOI:

10.1109/ULTSYM.2008.0234

Publication date:

2008

Document Version

Publisher's PDF, also known as Version of record

Link back to DTU Orbit

Citation (APA):

Andresen, H., Nikolov, S., Pedersen, M. M., Buckton, D., \& Jensen, J. A. (2008). Rocking convex array used for 3D synthetic aperture focusing. In 2008 IEEE Ultrasonics Symposium (Vol. 1-4, pp. 970-973). IEEE. I E E E International Ultrasonics Symposium. Proceedings https://doi.org/10.1109/ULTSYM.2008.0234

\section{General rights}

Copyright and moral rights for the publications made accessible in the public portal are retained by the authors and/or other copyright owners and it is a condition of accessing publications that users recognise and abide by the legal requirements associated with these rights.

- Users may download and print one copy of any publication from the public portal for the purpose of private study or research.

- You may not further distribute the material or use it for any profit-making activity or commercial gain

- You may freely distribute the URL identifying the publication in the public portal 


\title{
Rocking Convex Array used for 3D Synthetic Aperture Focusing
}

\author{
Henrik Andresen ${ }^{1,2}$, Svetoslav Ivanov Nikolov², Mads Møller Pedersen ${ }^{3}$, Daniel Buckton ${ }^{4}$ \\ and Jørgen Arendt Jensen ${ }^{1}$ \\ 1) Center for Fast Ultrasound Imaging, DTU-Elektro, \\ Bldg. 348, Technical University of Denmark, DK-2800 Lyngby, Denmark. \\ 2) B-K Medical, Mileparken 34, DK-2730 Herlev, Denmark. \\ 3) Department of Radiology, Rigshospitalet, Blegdamsvej 9, DK-2100 Copenhagen, Denmark \\ 4) GE Medical Systems, Kretztechnik, 4871 Zipf, Austria.
}

\begin{abstract}
Volumetric imaging can be performed using 1D arrays in combination with mechanical motion. Outside the elevation focus of the array, the resolution and contrast quickly degrade compared to the azimuth plane, because of the fixed transducer focus. The purpose of this paper is to use synthetic aperture focusing (SAF) for enhancing the elevation focusing for a convex rocking array, to obtain a more isotropic point spread function.

This paper presents further development of the SAF method, which can be used with curved array combined with a rocking motion. The method uses a virtual source (VS) for defocused multi-element transmit, and another VS in the elevation focus point. This allows a direct time-of-flight (ToF) to be calculated for a given 3D point. The method is evaluated using simulations from Field II and by measurements using the RASMUS experimental scanner with a $4.5 \mathrm{MHz}$ convex array (GE Kretztechnik, Zipf, Austria). The array has an elevation focus at $60 \mathrm{~mm}$ of depth, and the angular rocking velocity is up to $140 \%$ s. The scan sequence uses an $f_{\text {prf }}$ of $4500-7000 \mathrm{~Hz}$ allowing up to $15 \mathrm{~cm}$ of penetration. The full width at half max (FWHM) and main-lobe to side-lobe ratio (MLSL) is used as quantitative measurements.

The elevation FWHM for simulated scatterers placed at depths of 30 to $140 \mathrm{~mm}$ of depth were improved by $26.4 \%$ on average, and the MLSL ratio was improved by an average of $8.49 \mathrm{~dB}$ for the scatterers using 3D SA focusing. The elevation FWHM for a measured wire phantom was improved by $33.8 \%$ on average by applying 3D SA focusing. In-Vivo measurements show an improvement in $\mathrm{C}$-scans matching what is found in simulations and wire phantoms.

The method has shown the ability to improve the elevation focus and contrast for a convex rocking array. This was shown for simulations and for phantom and In-Vivo measurements using commercially available equipment.
\end{abstract}

\section{INTRODUCTION}

Rocking convex linear arrays are used to acquire 3D volume ultrasound (US) images. These arrays are available from commercial manufacturers, and many commercial scanners are able to use them them to gather a 3D dataset. These arrays are commonly used for abdominal scans to visualize organs and fetuses. The $3 \mathrm{D}$ volumes are created by imaging slices and stacking these to a volume. The volume has a good lateraldepth resolution, but poor elevation resolution because of the small elevation aperture and the fixed lens focus.
Using synthetic aperture (SA) focusing technique to improve the resolution of a fixed focus transducer has been shown feasible in [1]. This has further been used in [2][4] with linear and phased array transducers, to allow for both lateral and elevation focusing. Here a set of planes are beamformed with SA focusing, generating a set of lateral scanlines. The volume is created by beamforming the scan-lines in elevation by assuming that the elevation focus is a virtual source. Previous work has shown a significant increase in both elevation resolution, contrast, and signal to noise ratio (SNR) when applying this 2-step elevation beamforming method.

This paper presents the implementation of a 3D time of flight (ToF) calculation on a rocking convex array. The ToF method has previously been used for improving the elevation resolution for a translated linear array [5]. The mothod allows for a precise ToF calculation for lateral and elevation beamforming, using only a single beamforming step. This removes the requirement for beamforming in-plane points at the Nyquist criteria, and only uses points required for the volume. Section II will describe the equations and theory used in the method, the measurement setup is described in Section III, and Section IV will show the results from both simulation and measurements. The paper is concluded in Section V.

\section{THEORY}

SA focusing used from linear arrays is traditionally done by calculating the ToF for a spherical wave emitted by a single element, allowing each emission to contribute to the focusing of the entire insonified region. As a single element emissions gives a low SNR, virtual sources (VS) [3], [6], [7] and frequency modulation [8] are used to increase the emitted energy.

The method presented in [5] describes the ToF calculation for a given 3D-point for a linear array. This method has been implemented for a convex rocking array. To allow for a calculation using the rocking array, the ToF will be calculated using a rotated coordinate system. $[x, y, z]$ will denote the lateral, elevation, and depth direction, respectively. Two rotations are performed to place the emitting virtual source at the origo in the $x-y$ direction with the propagation direction along the 
$z$ axis. The first rotation is to counter the rocking motion, changing coordinates by

$$
\hat{r}_{p}=\left(\vec{r}_{p}-\vec{v}_{\text {ele,origo }}\right) \cdot \mathbf{M}(\phi)+\vec{v}_{\text {ele,origo }},
$$

where $\vec{r}_{p}$ is the point of interest, $\hat{r}_{p}$ is the rotated point, $\vec{v}_{\text {ele,origo }}$ is the point of rotation for the elevation rocking motion, and $\mathbf{M}(\phi)$ is the rotation matrix around the $x$-axis, which is dependent on the tilt of the array in the elevation direction. The second rotation is done by

$$
\tilde{r}_{p}=\left(\hat{r}_{p}-\vec{v}_{\text {lat,origo }}\right) \cdot \mathbf{M}(\theta)+\vec{v}_{\text {lat,origo }},
$$

where $\hat{r}_{p}$ is the point of interest rotated in the $y-z$ plane, $\tilde{r}_{p}$ is the final rotated point, $\vec{v}_{\text {lat,origo }}$ is the point of origin for the convex array curvature, and $\mathbf{M}(\theta)$ is the rotation matrix along the rotated $y$-axis, where $\theta$ is equal to the angle between the center of the array and the virtual transmit source.

The process of calculating the $\mathrm{ToF}$ is a two-fold process, as a VS is used both in the lateral and the elevation direction. The process is shown in Fig. 1, using the rotated coordinate system, where the point $\tilde{r}_{p}$ is the desired beamformed point, the dotted lines in the $x-z$ plane show the acceptance angle for the transmit VS and the dashed lines show the acceptance angle for the VS placed at the elevation focus. The point $\tilde{r}_{p}$ is projected onto the $x-z$-plane by letting the depth of the point be the distance traveled by the sound on a plane orthogonal to the $x-z$-plane, by placing a VS at the elevation focus on the same lateral position as $\tilde{r}_{p}$. The depth of the new point will be given by

$$
z_{\text {proj }}=\sqrt{\tilde{r}_{p, y}^{2}+\left(\tilde{r}_{p, z}-\tilde{z}_{\text {ele }}\right)^{2}} \cdot \operatorname{sign}\left(\tilde{r}_{p, z}-z_{\text {ele }}\right)+z_{\text {ele }},
$$

where $\tilde{r}_{p, y}, \tilde{r}_{p, z}$ is the elevation and depth position of $\tilde{r}_{p}$ relative to the transducer and $z_{\text {ele }}$ is the depth of the elevation focus. This virtual point is used for the ToF calculation using in-plane SA focusing. The virtual point, denoted $\vec{r}_{v}$, will have the coordinates $\left(r_{p, x}, 0, z_{e l e}\right)$. The equation for the total ToF for a transmission to the $m$ 'th receive element is given by

$$
t_{\mathrm{ToF}, m}=\frac{\left|\tilde{r}_{v}-\tilde{r}_{V S}\right|+\left|\tilde{r}_{v}-\tilde{r}_{r c v, m}\right|}{c},
$$

where $\tilde{r}_{V S}$ is the position of the transmit VS, $\tilde{r}_{r c v, m}$ is the position of the $m^{\prime}$ th receiving element, and $c$ is the speed of sound. The path is shown by the solid black line in Fig. 1 . The signal amplitude for a single point is given by summing the received signals at the time instances calculated by (4), which yields

$$
s\left(\vec{r}_{p}\right)=\sum_{m=1}^{M} \sum_{n=1}^{N} a_{m, n} \cdot g_{m, n}\left(t_{T o F, m}\right),
$$

where $a_{m, n}$ is the apodization and $g_{m, n}$ is the signal for the $m$ 'th receive channel of the $n$ 'th emission. $M$ is the number of receive elements and $N$ is the number of transmit VS's..

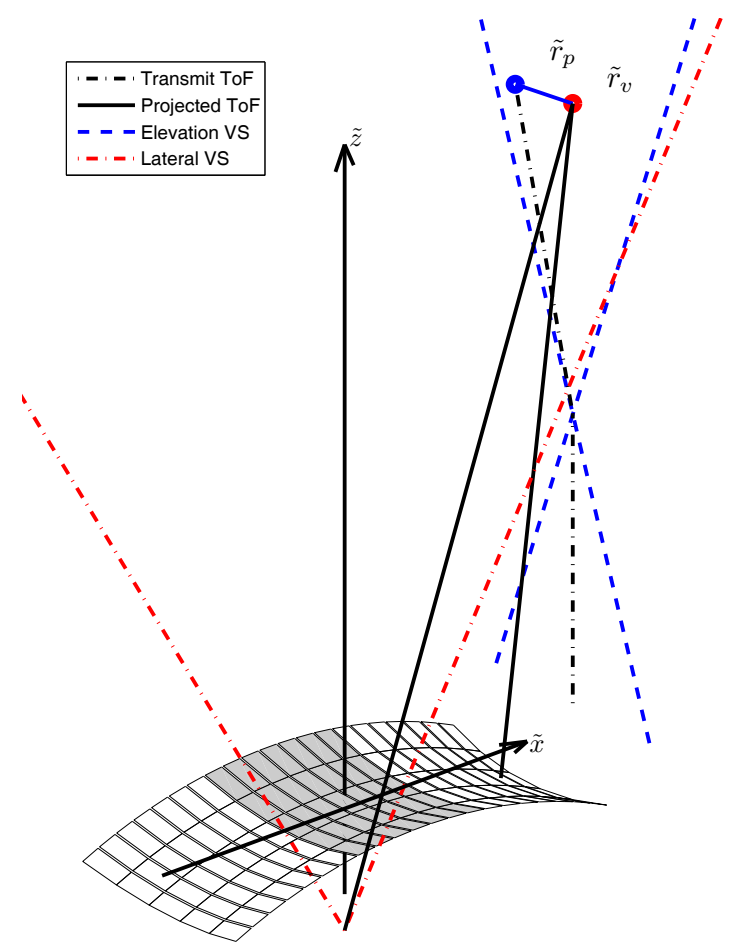

Figure 1. Illustration of the ToF calculation. Red dotted lines are transmit VS acceptance angle, blue dashed lines are elevation focus acceptance angle, the dotted black line is transmit ToF for the beamformed point, and solid black line is the total ToF for the projectet point. The blue point $\vec{r}_{p}$ is the desired beamformed point, and the red point $\vec{r}_{v}$ is the virtual projected point.

\section{Measurement Setup}

All measurements were done with the RASMUS experimental scanner available at the Center for Fast Ultrasound imaging (CFU). RASMUS is an abbreviation for Remotely Accessible Software configurable Multi-channel Ultrasound Sampling system, and was designed as a very flexible US system capable of transmitting arbitrary waveforms and storage of raw array channel data. A more detailed description is found in [9]. The transducer used is a convex array with a stepping motor to allow for a continuous back and forth rocking motion. The rocking system is controlled from a separate control box. A synchronization is attained by measuring a trigger signal emitted from the control box on the RASMUS system. Different measurements parameters are used, and will be referred to by a number in Table II. The transducer parameters are the same for all measurements and simulations and given in Table I. The parameters and the movement profile for the simulations are taken from a similar measurements on the RASMUS system.

\section{RESUlTS}

To evaluate the methods ability to improve elevation resolution, the full width at half max (FWHM) and main-lobe to side-lobe ratio (MLSL) is calculated for a set of simulated scatterers and for measurements performed on a wire phantom. The main-lobe width used to calculate the MLSL is defined at 
Table I

TRANSDUCER PARAMETERS.

\begin{tabular}{ll}
\hline Number of transducer elements & 128 \\
Center frequency, $f_{0}$ & $4.4 \mathrm{MHz}$ \\
Transducer element pitch angle & 0.268 \\
Transducer element height & $11 \mathrm{~mm}$ \\
Elevation focus & $62 \mathrm{~mm}$ \\
Convex curvature radius & $38.9 \mathrm{~mm}$ \\
Rocking radius & $22.6 \mathrm{~mm}$ \\
\hline
\end{tabular}

Table II

MEASUREMENT PARAMETERS.

\begin{tabular}{llll}
\hline Scan number & 1 & 2 & 3 \\
\hline Elements in virtual source & 7 & 7 & 7 \\
Emissions for full STA & 80 & 80 & 80 \\
Lateral VS Focusing F\# & $-\frac{1}{2}$ & $-\frac{1}{2}$ & $-\frac{1}{2}$ \\
FM-Chirp length & $15^{2} \mu \mathrm{s}$ & $15^{\mu} \mu \mathrm{s}$ & $15^{\mu} \mathrm{s}$ \\
Scan depth & $160 \mathrm{~mm}$ & $100 \mathrm{~mm}$ & $145^{\circ}$ \\
Receive apodization & Hamming & Hamming & Hamming \\
Receive F\# & 2 & 2 & 2 \\
Rocking sector & $35^{\circ}$ & $35^{\circ}$ & $35^{\circ}$ \\
$\mathrm{f}_{\text {prf }}$ & 4500 & 7000 & 5000 \\
Volumes per second & 3.5 & 3.8 & 3.5 \\
\hline
\end{tabular}

$-20 \mathrm{~dB}$ for the PSF created with 3D SA focusing. In addition, a $\mathrm{C}$-scan of an In-Vivo measurement is shown with and without 3D SA focusing.

The simulation is performed using the scan number 1 parameters in Table II. Fig. 2 shows the FWHM for a set of simulated scatterers between $30 \mathrm{~mm}$ and $140 \mathrm{~mm}$ of depth, which shows the methods ability to synthesize a larger aperture in the elevation direction. The improvement in FWHM is on average $26.4 \%$. The method is not able to synthesize a constant $\mathrm{F}^{\#}$ regardsless of depth, which is assumed to be caused by the high physical $\mathrm{F}^{\#}$ of the transducer as well as the rocking motion. Fig. 3 shows the MLSL for the same simulated scatterers. The gain in MLSL is $8.49 \mathrm{~dB}$ on average. Fig. 4 shows the projected PSF at a depth of $80 \mathrm{~mm}$. The

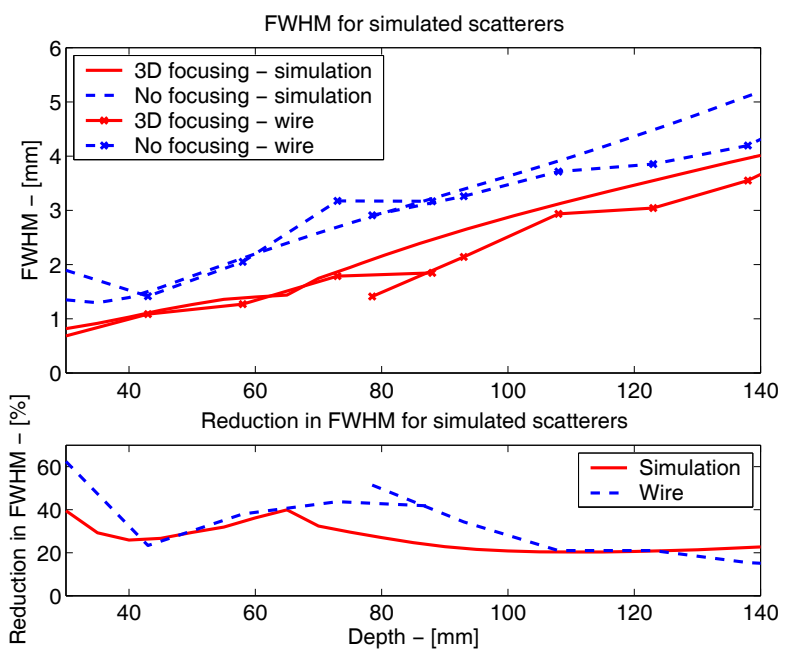

Figure 2. FWHM for simulated scatterers and measured wires with and without 3D SA focusing. The gain is calculated for both the measured and simulated scatterers.

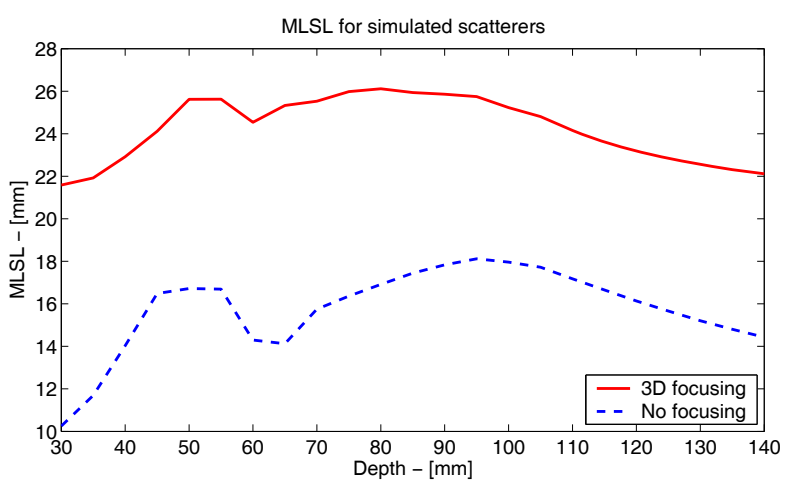

Figure 3. MLSL for simulated scatterers with and without 3D SA focusing.

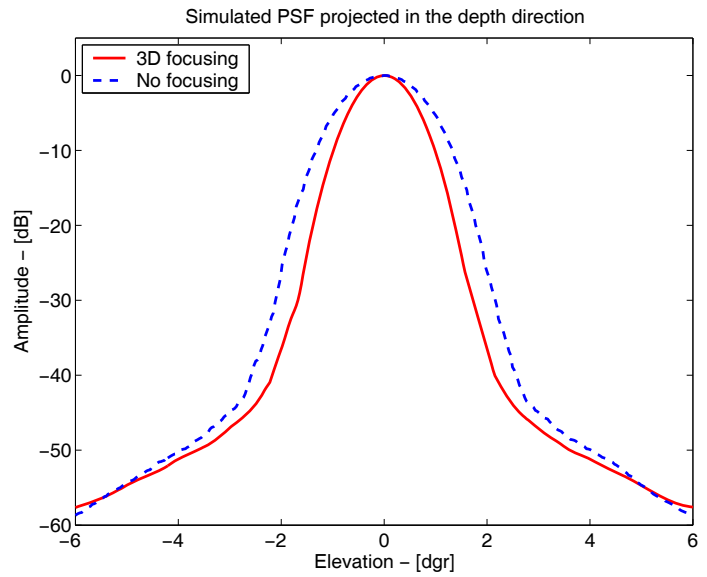

Figure 4. Simulated PSF at $80 \mathrm{~mm}$ of depth with and without 3D SA focusing.

mehod can be seen to improve the width of the PSF for all levels down to appr. $-50 \mathrm{~dB}$, in which the PSF without 3D SA focusing has slightly lower side-lobes.

A wire phantom was measured twice at different depths. The measurements are performed using the parameters from scan number 1 and 2, found in Table II. The two measurements are combined even though there is a change in the $f_{\text {prf }}$. Fig. 2 shows the FWHM for two measurements of a wire phantom on top of the results from the simulated scatterers. The evolution of the FWHM is very similar to the simulated scatterers, and the FWHM is almost the same value. The average improvement in FWHM for the measured scatterers is $33.8 \%$, and is overall very similar to the simulated result.

Fig. 5 show the measured PSF in the elevation direction. The PSF is slightly smaller in width, but is otherwise comparable to the simulated PSF. The results shows a good correlation between the simulated and measured response, which validates the methods use for equipment readily available from commercial systems.

The C-scan of an In-Vivo measurement is shown in Fig. 6 and 7 . The scan is showing the liver of a healthy 26 year old male volunteer. To the right in the middle is a vein, but of most interest is the speckle in the liver. This clearly shows a reduction in the elevation direction, confirming the simulation 


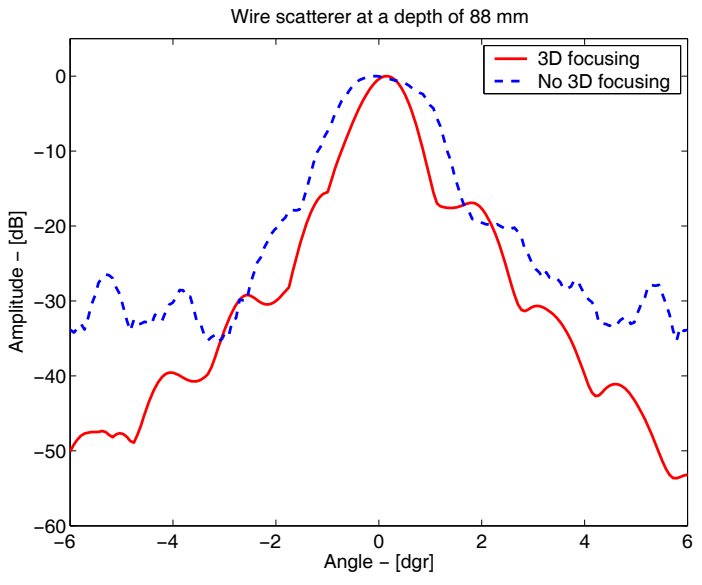

Figure 5. Measured PSF at $88 \mathrm{~mm}$ of depth with and without 3D SA focusing.

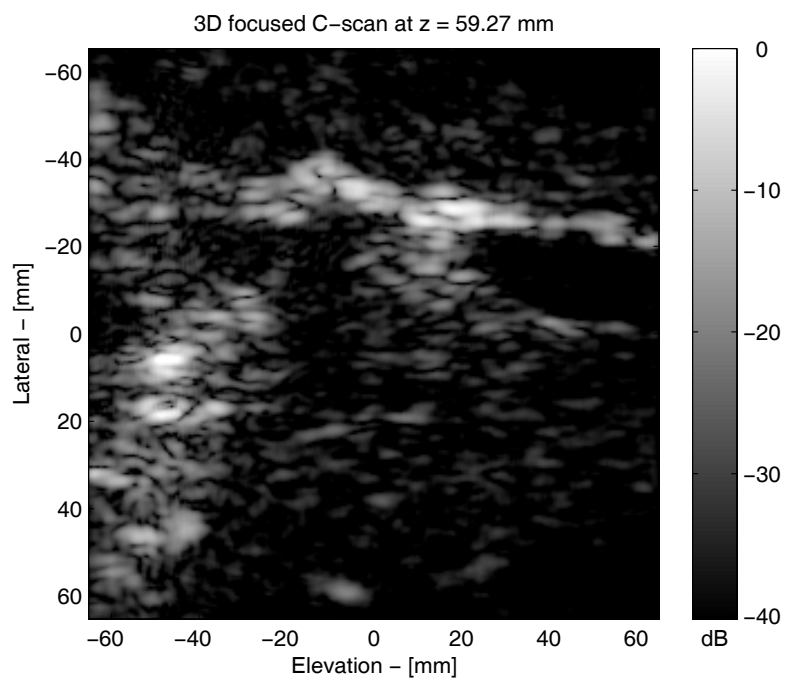

Figure 6. In-Vivo measurement of liver in a healthy 26 year old male volunteer.

and wire phantom measurements.

\section{CONClusion}

The method for 3D synthetic aperture focusing has been successfully implemented for a rocking convex array. The method is not able to maintain a constant $\mathrm{F}^{\#}$, but shows an average improvement to the FWHM of $26.4 \%$ for simulated scatterers and $33.8 \%$ for a measured wire phantom. The simulated scatterers show a reduction in the width of the PSF between $0 \mathrm{~dB}$ and $-50 \mathrm{~dB}$, after which the amplitude is comparable. A C-scan of an In-Vivo measurement also shows the expected inprovement in the elevation direction.

The results presented in this paper proves the viability of the method to improve the contrast and resolution of real-time $3 \mathrm{D}$ ultrasound data acquisition using commercially available equipment.

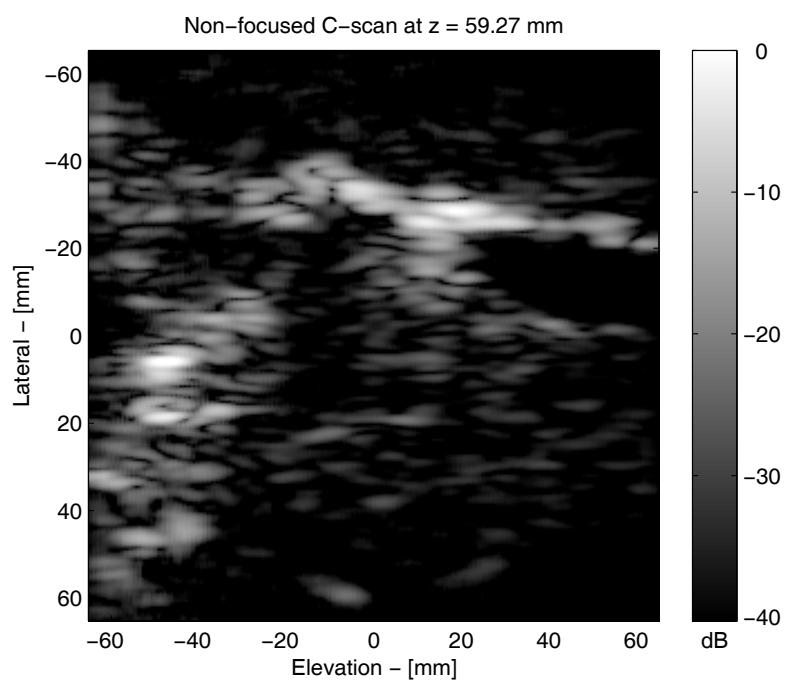

Figure 7. In-Vivo measurement of liver in a healthy 26 year old male volunteer.

\section{ACKNOWLEDGMENT}

This work was supported by grant 71122 from the Danish Ministry of Science, Technology and Innovation, grant 9700883, 9700563 and 26-04-0024 from the Danish Science Foundation and by B-K Medical Aps., Denmark.

\section{REFERENCES}

[1] C. H. Frazier and W. D. O'Brien. Synthetic aperture techniques with a virtual source element. IEEE Trans. Ultrason., Ferroelec., Freq. Contr., 45:196-207, 1998.

[2] S. I. Nikolov and J. A. Jensen. 3D synthetic aperture imaging using a virtual source element in the elevation plane. In Proc. IEEE Ultrason. Symp., volume 2, pages 1743-1747, 2000.

[3] S. I. Nikolov. Synthetic aperture tissue and flow ultrasound imaging. $\mathrm{PhD}$ thesis, Ørsted•DTU, Technical University of Denmark, 2800, Lyngby, Denmark, 2001.

[4] S. I. Nikolov, P. Santén, O. Bjuvsten, and J. A. Jensen. Parameter study of 3D synthetic aperture post-beamforming procedure. Ultrasonics, 44:e159-e164, 2006.

[5] H. Andresen, S. I. Nikolov, and J. A. Jensen. Precise time-of-flight calculation for 3D synthetic aperture focusing. In Proc. IEEE Ultrason. Symp., pages 224-227, 2007.

[6] M. Karaman, P. C. Li, and M. O’Donnell. Synthetic aperture imaging for small scale systems. IEEE Trans. Ultrason., Ferroelec., Freq. Contr. 42:429-442, 1995.

[7] S. I. Nikolov and J. A. Jensen. Virtual ultrasound sources in highresolution ultrasound imaging. In Proc. SPIE - Progress in biomedical optics and imaging, volume 3, pages 395-405, 2002.

[8] F. Gran and J. A. Jensen. Designing waveforms for temporal encoding using a frequency sampling method. IEEE Trans. Ultrason., Ferroelec., Freq. Contr., 54(10):2070-2081, October 2007.

[9] J. A. Jensen, O. Holm, L. J. Jensen, H. Bendsen, S. I. Nikolov, B. G. Tomov, P. Munk, M. Hansen, K. Salomonsen, J. Hansen, K. Gormsen, H. M. Pedersen, and K. L. Gammelmark. Ultrasound research scanner for real-time synthetic aperture image acquisition. IEEE Trans. Ultrason., Ferroelec., Freq. Contr., 52 (5):881-891, May 2005. 